\title{
Synthesis, Characterization and Cytotoxic Activity of Tioconazole Coordination Compounds with Nickel(II), Palladium(II) and Platinum(II)
}

\author{
Carmela Crisóstomo-Lucas ${ }^{1}$, Rubí Navarro-Peñaloza ${ }^{1}$, Naytzé Ortiz-Pastrana ${ }^{1,2}$, Francisco Sánchez- \\ Bartéz ${ }^{1,3}$, Isabel Gracia-Mora ${ }^{1,3}$, Norah Barba-Behrens ${ }^{1 *}$ \\ ${ }^{1}$ Departamento de Química Inorgánica, Facultad de Química, Universidad Nacional Autónoma de México, \\ Ciudad Universitaria, Coyoacán, 04510, Ciudad de México, México. \\ ${ }^{2}$ Departamento de Química, Cinvestav, AP 14-740, 07000, Ciudad de México, México. \\ ${ }^{3}$ Unidad de Investigación Preclínica (UNIPREC), Facultad de Química, Universidad Nacional Autónoma de \\ México, Ciudad de México, México.
}

* Corresponding author: Norah Barba-Behrens, norah@unam.mx

Received May 11 ${ }^{\text {th }}, 2018$; Accepted December 11 ${ }^{\text {th }}, 2018$.

DOI: http://dx.doi.org/10.29356/jmcs.v62i4.563

\begin{abstract}
Coordination compounds of nickel(II), palladium(II) and platinum(II) with tioconazole (tcnz) were synthesized and characterized by infrared, UV-Vis-NIR, elemental analysis, molar conductivity, magnetic susceptibility, mass spectrometry, NMR spectroscopy and X-ray diffraction. Tioconazole presented a monodentate coordination mode, through the nitrogen atom of the imidazolic ring. The nickel(II) compounds stabilized octahedral geometries. For the $\left[\mathrm{Ni}(\operatorname{tcnz})_{2}\left(\mathrm{NO}_{3}\right)_{2}\right] \cdot \mathrm{H}_{2} \mathrm{O}$ compound, the nitrate anion present a bidentate coordination mode, while for the dinuclear $\left[\mathrm{Ni}(\mathrm{tcnz})_{2}(\mathrm{OAc})_{2}\right]_{2} \cdot 3 \mathrm{H}_{2} \mathrm{O}$ compound, the acetate groups behave as bridging ligands. When different molar ratios of the corresponding nickel(II) halides were used on the reaction synthesis, three tcnz ligands in the $\left[\mathrm{Ni}(\operatorname{tcnz})_{3} \mathrm{Br}_{2}\left(\mathrm{H}_{2} \mathrm{O}\right)\right]$, or six in $\left[\mathrm{Ni}(\mathrm{tcnz})_{6}\right] \mathrm{Cl}_{2}$ and [Ni(tcnz) $)_{\mathrm{Br}_{2}}$, are coordinated to the nickel(II) atom. The palladium(II) and platinum(II) compounds, $\left[\mathrm{Pd}(\mathrm{tcnz})_{2} \mathrm{Cl}_{2}\right], \quad\left[\mathrm{Pt}(\mathrm{tcnz})_{2} \mathrm{Cl}_{2}\right] \cdot 2 \mathrm{H}_{2} \mathrm{O}$ and $\left[\mathrm{Pd}(\mathrm{tcnz})_{2}(\mathrm{OAc})_{2}\right]$, presented a square planar geometry. The $\left[\mathrm{Ni}(\mathrm{tcnz})_{6}\right] \mathrm{X}_{2}\left(\mathrm{X}^{-}=\mathrm{Cl}, \mathrm{Br}\right)$ compounds stabilized 3D supramolecular arrangements through hydrogen bonding and $\pi \cdots \pi$ stacking interactions, between benzene rings of neighboring molecules. The in vitro cytotoxic activity of the synthesized compounds was studied in four different human carcinoma cell lines: HCT-15 (colon), HeLa (cervix-uterine), MCF-7 (breast) and PC-3 (prostate).
\end{abstract}

Keywords: Tioconazole; coordination compounds; nickel(II); palladium(II); platinum(II); intermolecular interactions; cytotoxic activity.

Resumen. Los compuestos de coordinación de níquel(II), paladio(II) y platino(II) con tioconazol (tcnz) fueron sintetizados y caracterizados por infrarrojo, UV-Vis-NIR, análisis elemental, conductividad molar, susceptibilidad magnética, espectrometría de masas, espectroscopía de RMN y difracción de rayos X. El tioconazol presenta un modo de coordinación monodentado, a través del átomo de nitrógeno del anillo de imidazol. Los compuestos de coordinación de niquel(II) estabilizan geometrías octaédricas. En el compuesto $\left[\mathrm{Ni}(\mathrm{tcnz})_{2}\left(\mathrm{NO}_{3}\right)_{2}\right] \cdot \mathrm{H}_{2} \mathrm{O}$, el anión nitrato presenta un modo de coordinación bidentado, mientras que en el compuesto dinuclear $\left[\mathrm{Ni}(\mathrm{tcnz})_{2}(\mathrm{OAc})_{2}\right]_{2} \cdot 3 \mathrm{H}_{2} \mathrm{O}$, los acetatos se comportan como ligantes puente. Empleando diferentes relaciones molares en las reacciones de síntesis de los correspondientes haluros de niquel(II) se obtuvieron tres ligantes tenz coordinados al átomo metálico en el compuesto [ $\left.\mathrm{Ni}(\operatorname{tcnz})_{3} \mathrm{Br}_{2}\left(\mathrm{H}_{2} \mathrm{O}\right)\right]$, o seis en los compuestos $\left[\mathrm{Ni}(\mathrm{tcnz})_{6}\right] \mathrm{Cl}_{2}$ y $\left[\mathrm{Ni}(\mathrm{tcnz})_{6}\right] \mathrm{Br}_{2}$. Los complejos de paladio(II) y platino(II), [ $\mathrm{Pd}(\mathrm{tcnz})_{2} \mathrm{Cl}_{2}$, $\left[\mathrm{Pt}(\operatorname{tcnz})_{2} \mathrm{Cl}_{2}\right] \cdot 2 \mathrm{H}_{2} \mathrm{O}$ y $\left[\mathrm{Pd}(\mathrm{tcnz})_{2}(\mathrm{OAc})_{2}\right]$, presentan una geometría cuadrada plana. Los compuestos $\left[\mathrm{Ni}(\mathrm{tcnz})_{6}\right] \mathrm{X}_{2}\left(\mathrm{X}^{-}=\mathrm{Cl}, \mathrm{Br}\right)$ estabilizan arreglos supramoleculares en $3 \mathrm{D}$, a través interacciones de puentes de hidrógeno y apilamientos $\pi \cdots \pi$ entre los anillos bencénicos de moléculas vecinas. La actividad citotóxica in 
vitro de los compuestos sintetizados fue estudiada en cuatro diferentes líneas celulares de carcinoma humano: HCT-15 (colon) y HeLa (cervicouterino), MCF-7 (mama) and PC-3 (próstata).

Palabras clave: Tioconazol; compuestos de coordinación; níquel(II); paladio(II); platino(II); interacciones intermoleculares; actividad citotóxica.

\section{Introduction}

The discovery of active sites containing nickel centers in metalloproteins, [1-4] has stimulated the study of its coordination compounds and their biological activity, such as antitumor, anticonvulsants or antiepileptic, antibacterial and antifungal.[5,6] Additionally, palladium and platinum, also from group 10, have shown anticancer activity.[7-10] Platinum(II) compounds, as cisplatin, oxaliplatin and carboplatin, are the more effective anticancer drugs used in chemotherapy.[11,12]

Cisplatin, used for the testicular and ovarian cancer treatment, is one of the most widely used antitumor drugs in the world.[13-15] However, it has side effects such as nephrotoxicity, drug tolerance, limited solubility and intravenous administration.[14,16-19] Due to the fact that the cancer is the second leading cause of death, $[16,20,21]$ the development of improved metal based drugs is currently of interest.

When designing new antitumor agents, the similarity in the chemistry of the $\mathrm{Pt}^{\mathrm{II}}$ and $\mathrm{Pd}^{\mathrm{II}}$ compounds has led to the study of palladium antitumor drugs with high activity.[22-26] Some of them are stabilized by chelates, as Schiff bases, or by voluminous ligands with monodentate nitrogen atoms,[22] which presented activity in HeLa cell line. It has been reported that trans-Pd compounds with Schiff base ligands have better activity than the cis-Pd compounds.[18,22] Additionally, a series of coordination compounds whit benzylamine and $\mathrm{Pd}^{\mathrm{II}}$ were studied on MCF-7 and MDA-MB-231 breast cancer cell lines, indicating an effective anticancer potential. [17]

When combining metal ions with an established biological activity of the ligands, an enhancement or a modification of their pharmacological properties has been observed, as it is the case of ticonazole, clotrimazole and miconazole, with antifungal properties, which their organometallic ruthenium(II) compounds showed antiparasite activity.[27] Transition metal coordination compounds with Shiff base derivatives, $\left(\mathrm{Co}^{\mathrm{II}}, \mathrm{Ni}^{\mathrm{II}}, \mathrm{Cu}^{\mathrm{II}}, \mathrm{Zn}^{\mathrm{II}}\right)$ have proved to enhance the antimicrobial and antifungal activity of the free ligands.[28]

In a previous study, we synthesized and characterized $\mathrm{Cu}^{\mathrm{II}}$ and $\mathrm{Zn}^{\mathrm{II}}$ coordination compounds with tioconazole (tcnz) and their cytotoxic activity in HCT-15 and HeLa cell lines was studied. The octahedral $\left[\mathrm{Cu}(\mathrm{tcnz})_{4} \mathrm{Cl}_{2}\right]$ compound showed promising activity in the HCT-15 cell line. While the tetrahedral [ $\mathrm{Zn}(\operatorname{tcnz})_{2} \mathrm{Br}_{2}$ ] compound had cytotoxic activity in HeLa cell lines.[29]

Continuing our work in this field, $\mathrm{Ni}^{\mathrm{II}}, \mathrm{Pt}^{\mathrm{II}}$ and $\mathrm{Pd}^{\mathrm{II}}$ compounds were synthesized and characterized (Fig. 1) as their biological activity as possible anticancer agents in HCT-15, HeLa, MCF-7 and PC-3 cell lines, was studied. 


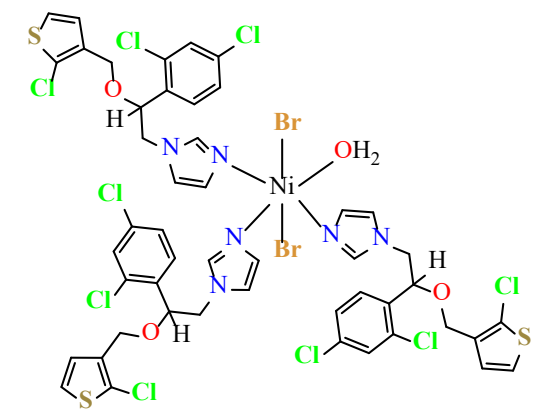

$\left[\mathrm{Ni}(\operatorname{tcnz})_{3}(\mathrm{Br})_{2}\left(\mathrm{H}_{2} \mathrm{O}\right)\right]$

(1)

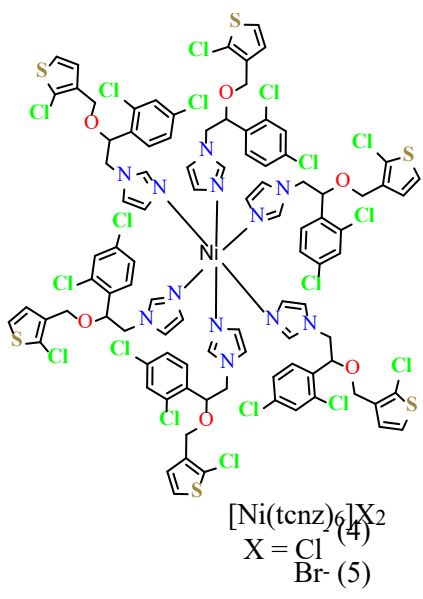<smiles>Clc1ccc(C(Cn2ccnc2)OCc2ccsc2Cl)c(Cl)c1</smiles>

Tioconazole (tcnz)

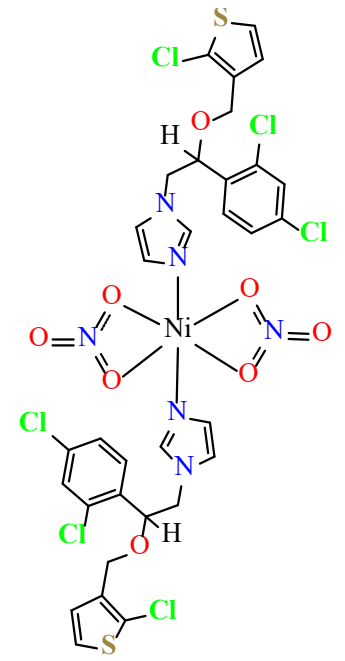

$\left[\mathrm{Ni}(\operatorname{tcnz})_{2}\left(\mathrm{NO}_{3}\right)_{2}\right] \cdot \mathrm{H}_{2} \mathrm{O}$

(2)

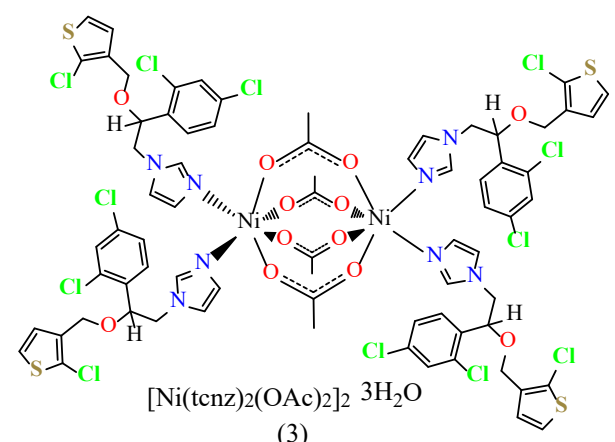

Fig. 1. Structures for the tioconazole coordination compounds with $\mathrm{Ni}^{\mathrm{II}}, \mathrm{Pd}^{\mathrm{II}}$ and $\mathrm{Pt}^{\mathrm{II}}$.

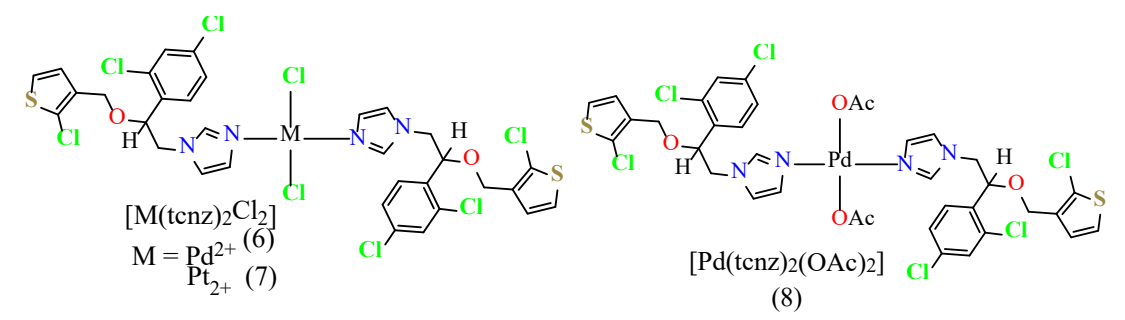




\section{Experimental}

\section{Materials and methods}

All reagents and solvents were purchased and used without any further purification: tioconazole $98 \%$ (Aldrich, Co); $\mathrm{NiCl}_{2} \cdot 6 \mathrm{H}_{2} \mathrm{O}, \mathrm{NiBr}_{2} \cdot 3 \mathrm{H}_{2} \mathrm{O}, \mathrm{Ni}\left(\mathrm{NO}_{3}\right)_{2} \cdot 6 \mathrm{H}_{2} \mathrm{O}, \mathrm{Ni}(\mathrm{OAc})_{2} \cdot 4 \mathrm{H}_{2} \mathrm{O}$ (J.T. Baker); $\mathrm{PdCl}_{2}, \mathrm{PtCl}_{2}$ and $\mathrm{Pd}(\mathrm{OAc})_{2}$ (Aldrich, Co); solvents (Merck). The complexes $\left[\mathrm{PdCl}_{2}\left(\mathrm{CH}_{3} \mathrm{CN}\right)_{2}\right]$ and $\left[\mathrm{PtCl}_{2}\left(\mathrm{CH}_{3} \mathrm{CN}\right)_{2}\right]$ were synthesized according with the reported procedure.[30] FT IR spectra in the range 4000-400 $\mathrm{cm}^{-1}$ were collected in a Perkin Elmer FT-IR Spectrum 400 spectrophotometer with an universal ATR sampling accessory at $298 \mathrm{~K}$. Mass spectra (MS-ESI ${ }^{+}$) were determined in an Esquire 6000 mass spectrometer (Fig. S1S6). Elemental analyses for carbon, hydrogen, nitrogen and sulfur were carried out with a Fisons EA 1108 analyzer. Magnetic susceptibility measurements at room temperature of powdered samples were obtained on a Johnson-Matthey DG8 5HJ balance, using the Gouy method. NMR spectra were obtained at room temperature on a $300 \mathrm{MHz}$ Bruker-Avance Unity spectrometer. ${ }^{1} \mathrm{H}$ and ${ }^{13} \mathrm{C}\left\{{ }^{1} \mathrm{H}\right\}$ NMR spectra were obtained using DMSO- $d_{6}$ and $\mathrm{CDCl}_{3}$ (Fig. S7-S15). Electronic spectra were measured over the range $40000-5000 \mathrm{~cm}^{-1}$ by the diffuse reflectance method on a Cary-5000 Varian spectrophotometer at $298 \mathrm{~K}$ (Fig. S16).

\section{Synthesis of the coordination compounds}

Coordination compounds form the $\mathrm{Ni}^{\mathrm{II}}$ salts were synthesized by similar procedures. A solution of one equivalent of the corresponding transition metal salt in acetone was added to a solution of one equivalent of tioconazole in acetone, with exception of the $\left[\mathrm{Ni}(\operatorname{tcnz})_{2}(\mathrm{OAc})_{2}\right]_{2} \cdot 3 \mathrm{H}_{2} \mathrm{O} 3$ compound where ethanol was used as solvent. For the compounds $\left[\mathrm{Ni}(\mathrm{tcnz})_{6}\right] \mathrm{Cl}_{2} 4$ and $\left[\mathrm{Ni}(\mathrm{tcnz})_{6}\right] \mathrm{Br}_{2} 5$ a 1:3 ratio was used. The reaction mixture was heated under reflux with constant stirring during $24 \mathrm{~h}$. $\mathrm{Pd}^{\mathrm{II}}$ and $\mathrm{P}^{\mathrm{II}}$ compounds were synthesized using a 1:2 ratio (metal salt:tenz) in acetone. The solvent was evaporated at RT and the products were washed with water and ethanol and dried under vacuum overnight.

\section{Synthesis of $\left[\mathrm{Ni}(\operatorname{tenz})_{3} \mathrm{Br}_{2}\left(\mathrm{H}_{2} \mathrm{O}\right)\right](1)$}

$\mathrm{NiBr}_{2} \cdot 3 \mathrm{H}_{2} \mathrm{O}(0.07 \mathrm{~g}, 0.25 \mathrm{mmol})$ was added to a solution of tioconazole $(0.1 \mathrm{~g}, 0.25 \mathrm{mmol})$ in acetone $(15 \mathrm{~mL})$, the obtained dark blue solution was left to stand at RT, a bright blue solid precipitated. UV-Vis-NIR $v\left(\mathrm{~cm}^{-1}\right): v_{1}=8665, v_{2}=14993$ and $v_{3}=24918$. FT-IR (ATR, $\left.v^{-1}\right): v(\mathrm{O}-\mathrm{H}) 3324, v(\mathrm{C}=\mathrm{N}) 1589, v(\mathrm{C}-\mathrm{O}-\mathrm{C})$ 1088, v(C-S) 736. MS-ESI ${ }^{+}(\mathrm{m} / \mathrm{z}) 1301\left[\mathrm{C}_{48} \mathrm{H}_{39} \mathrm{Cl}_{9} \mathrm{BrN}_{6} \mathrm{O}_{3} \mathrm{~S}_{3} \mathrm{Ni}^{+}\right.$. Anal. Found: C, 41.08; H, 2.51; N, 6.10; S, 5.99\%. Calc. for $\mathrm{C}_{48} \mathrm{H}_{41} \mathrm{Cl}_{9} \mathrm{Br}_{2} \mathrm{~N}_{6} \mathrm{O}_{4} \mathrm{~S}_{3} \mathrm{Ni}: \mathrm{C}, 41.19 ; \mathrm{H}, 2.95 ; \mathrm{N}, 6.00 ; \mathrm{S}, 6.87 \%$. $\mu_{\text {eff }}=3.40$ BM. Yield: $(0.19$ g, 92\%).

\section{Synthesis of $\left[\mathrm{Ni}(\operatorname{tenz})_{2}\left(\mathrm{NO}_{3}\right)_{2}\right] \cdot \mathrm{H}_{2} \mathrm{O}(2)$}

$\mathrm{Ni}\left(\mathrm{NO}_{3}\right)_{2} \cdot 6 \mathrm{H}_{2} \mathrm{O}(0.074 \mathrm{~g}, 0.25 \mathrm{mmol})$ was added to a solution of tioconazole $(0.1 \mathrm{~g}, 0.25 \mathrm{mmol})$ in acetone $(15 \mathrm{~mL})$. A dark green solution was observed, a green solid was obtained. UV-Vis-NIR $v\left(\mathrm{~cm}^{-1}\right): v_{1}=$ 8936, $v_{2}=15553$ and $v_{3}=25477$. FT-IR $\left(\mathrm{ATR}, v \mathrm{~cm}^{-1}\right): v(\mathrm{O}-\mathrm{H}) 3344, v(\mathrm{C}=\mathrm{N}) 1589, v_{\text {as }}\left(\mathrm{NO}_{3}\right) 1468, v_{\mathrm{s}}\left(\mathrm{NO}_{3}\right)$ 1306, v(N=O) 1235, v(C-O-C) 1087, v(C-S) 736. MS-ESI ${ }^{+}(\mathrm{m} / \mathrm{z}) 896\left[\mathrm{C}_{32} \mathrm{H}_{26} \mathrm{Cl}_{6} \mathrm{~N}_{5} \mathrm{O}_{5} \mathrm{~S}_{2} \mathrm{Ni}\right]^{+}$. Anal. Found: C, 39.36; H, 2.90; N, 8.45; S, 6.26\%. Calc. for $\mathrm{C}_{32} \mathrm{H}_{28} \mathrm{Cl}_{6} \mathrm{~N}_{6} \mathrm{O}_{9} \mathrm{~S} 2 \mathrm{Ni}$ : C, 39.37; H, 2.89; N, 8.61; $\mathrm{S}, 6.57 \%$. $\mu_{\mathrm{eff}}=$ 3.50 BM. Yield: $(0.11 \mathrm{~g}, 81 \%)$.

\section{Synthesis of $\left[\mathrm{Ni}(\operatorname{tenz})_{2}(\mathrm{OAc})_{2}\right]_{2} \cdot 3 \mathrm{H}_{2} \mathrm{O}(3)$}

$\mathrm{Ni}(\mathrm{OAc})_{2} \cdot 4 \mathrm{H}_{2} \mathrm{O}(0.064 \mathrm{~g}, 0.25 \mathrm{mmol})$ was added to a solution of tioconazole $(0.1 \mathrm{~g}, 0.25 \mathrm{mmol})$ in ethanol $(15 \mathrm{~mL})$. An emerald green solution was observed, a bright green solid precipitated. UV-Vis-NIR $v\left(\mathrm{~cm}^{-1}\right): v_{1}=8936, v_{2}=15417$ and $v_{3}=25409$. FT-IR (ATR, $\left.v \mathrm{~cm}^{-1}\right): v(\mathrm{O}-\mathrm{H}) 3128, v(\mathrm{C}=\mathrm{N}) 1586, v_{\text {as }}(\mathrm{COO})$ $1558, v_{\mathrm{s}}(\mathrm{COO}) 1408, v(\mathrm{C}-\mathrm{O}-\mathrm{C}) 1084, v(\mathrm{C}-\mathrm{S}) 732 . \mathrm{MS}^{-E_{S I}}(\mathrm{~m} / \mathrm{z}) 1456\left[\mathrm{C}_{54} \mathrm{H}_{47} \mathrm{Cl}_{9} \mathrm{~N}_{6} \mathrm{O}_{9} \mathrm{~S}_{3} \mathrm{Ni}_{2}\right]^{+}$. Anal. Found: C, 42.45; H, 3.01; N, 5.70; S, 5.35\%. Calc. for $\mathrm{C}_{36} \mathrm{H}_{38} \mathrm{Cl}_{6} \mathrm{~N}_{4} \mathrm{O}_{9} \mathrm{~S}_{2} \mathrm{Ni}: \mathrm{C}, 42.97 ; \mathrm{H}, 3.81 ; \mathrm{N}, 5.57 ; \mathrm{S}, 6.37 \%$. $\mu_{\text {eff }}$ $=3.20$ BM. Yield: $(0.11 \mathrm{~g}, 90 \%$. $)$.

\section{Synthesis of $\left[\mathrm{Ni}(\operatorname{tenz})_{6}\right] \mathrm{Cl}_{\mathbf{2}}$ (4)}

$\mathrm{NiCl}_{2} \cdot 6 \mathrm{H}_{2} \mathrm{O}(0.07 \mathrm{~g}, 0.30 \mathrm{mmol})$ was added to a solution of tioconazole $(0.34 \mathrm{~g}, 0.90 \mathrm{mmol})$ in acetone $(15 \mathrm{~mL})$, the obtained blue solution was left to stand at RT. After two weeks purple crystals suitable for X-ray diffraction were isolated. UV-Vis-NIR $v\left(\mathrm{~cm}^{-1}\right): v_{1}=10880, v_{2}=17550$ and $v_{3}=24330$. FT-IR 
(ATR, $\left.v \mathrm{~cm}^{-1}\right): v(\mathrm{C}=\mathrm{N}) 1589, v(\mathrm{C}-\mathrm{O}-\mathrm{C}) 1078, v(\mathrm{C}-\mathrm{S}) 738$. Due to its insolubility in common solvents it was not possible to obtain its $\mathrm{MS}^{-\mathrm{ESI}^{+}}(\mathrm{m} / \mathrm{z})$. Anal. Found: C, 7.70; H, 3.33; N, 6.58; S 7.70\%. Calc. for $\mathrm{C}_{96} \mathrm{H}_{78} \mathrm{Cl}_{20} \mathrm{~N}_{12} \mathrm{O}_{6} \mathrm{~S}_{6} \mathrm{Ni}$ : C, 46.94; H, 3.20; N, 6.84; S, 7.83\%. $\mu_{\text {eff }}=3.14$ BM. Yield: (0.24g, 33\%).

\section{Synthesis of $\left[\mathrm{Ni}(\operatorname{tenz})_{6}\right] \mathrm{Br}_{2}(5)$}

$\mathrm{NiBr}_{2} \cdot 3 \mathrm{H}_{2} \mathrm{O}(0.07 \mathrm{~g}, 0.25 \mathrm{mmol})$ was added to a solution of tioconazole $(0.3 \mathrm{~g}, 0.75 \mathrm{mmol})$ in acetone $(15 \mathrm{~mL})$, the obtained blue solution was let to stand at RT. After a week purple crystals suitable for Xray diffraction were isolated. UV-Vis-NIR $v\left(\mathrm{~cm}^{-1}\right): v_{1}=10880, v_{2}=17550$ and $v_{3}=24330$. FT-IR (ATR, $v$ $\left.\mathrm{cm}^{-1}\right): v(\mathrm{C}=\mathrm{N}) 1589, v(\mathrm{C}-\mathrm{O}-\mathrm{C}) 1079, v(\mathrm{C}-\mathrm{S}) 737$. Due to its insolubility in common solvents it was not possible to obtain its MS-ESI ${ }^{+}(\mathrm{m} / \mathrm{z})$. Anal. Found: C, 44.47; H, 3.21; N, 7.54; S, 6.59\%. Calc. for $\mathrm{C}_{96} \mathrm{H}_{78} \mathrm{Cl}_{18} \mathrm{Br}_{2} \mathrm{~N}_{12} \mathrm{O}_{6} \mathrm{~S}_{6} \mathrm{Ni}: \mathrm{C}, 45.31 ; \mathrm{H}, 3.09 ; \mathrm{N}, 7.56 ; \mathrm{S}, 6.60 \%$. $\mu_{\text {eff }}=3.01$ BM. Yield: $(0.25 \mathrm{~g}, 36 \%)$.

\section{Synthesis of $\left[\mathrm{Pd}(\operatorname{tenz})_{2} \mathrm{Cl}_{2}\right](6)$}

$\left[\mathrm{PdCl}_{2}\left(\mathrm{CH}_{3} \mathrm{CN}\right)_{2}\right](0.05 \mathrm{~g}, 0.19 \mathrm{mmol}$ was added to a solution of tioconazole $(0.14 \mathrm{~g}, 0.38 \mathrm{mmol})$ in acetone $(15 \mathrm{~mL})$ during $24 \mathrm{~h}$ at RT a light yellow solid precipitated from the solution. UV-Vis-NIR $v\left(\mathrm{~cm}^{-1}\right)$ : $v_{1}=25595$. FT-IR (ATR, $\left.v \mathrm{~cm}^{-1}\right): v(\mathrm{C}=\mathrm{N})$ 1587, $v(\mathrm{C}-\mathrm{O}-\mathrm{C}) 1084, v(\mathrm{C}-\mathrm{S}) 735 .{ }^{1} \mathrm{H}$ NMR $\left(\mathrm{DMSO}_{\mathrm{d}}, 300 \mathrm{MHz}\right)$ : $8.08(2 \mathrm{H}, \mathrm{s}, \mathrm{CH}, \mathrm{Ha}), 7.67(2 \mathrm{H}, \mathrm{d}, \mathrm{CH}, \mathrm{Hf}), 7.51(2 \mathrm{H}, \mathrm{dd}, \mathrm{CH}, \mathrm{Hh}), 7.43(2 \mathrm{H}, \mathrm{s}, \mathrm{CH}, \mathrm{Hj}), 7.41(2 \mathrm{H}, \mathrm{s}, \mathrm{CH}$, $\mathrm{Hc}), 7.17(2 \mathrm{H}, \mathrm{s}, \mathrm{CH}, \mathrm{Hg}), 7.11(2 \mathrm{H}, \mathrm{s}, \mathrm{CH}, \mathrm{Hb}), 6.89$ (2H, d, CH, Hk), $4.95(2 \mathrm{H}, \mathrm{dd}, \mathrm{CH}, \mathrm{He}), 4.37-4.26(8 \mathrm{H}$, $\mathrm{m}, \mathrm{CH}_{2}, \mathrm{Hi}, \mathrm{d}$ ). ${ }^{13} \mathrm{C}$ NMR (DMSO-d 6 , 75MHz assignments by HSQC ): 139.79 (C1), 134.44 (C6), 134.06 (C7), 133.73 (C9), 133.20 (C13), 129.36 (C14), 128.99 (C8), 128.63 (C10), 127.98 (C15), 127.91 (C11), 126.50

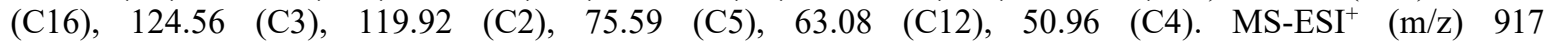
$\left[\mathrm{C}_{32} \mathrm{H}_{26} \mathrm{Cl}_{7} \mathrm{~N}_{4} \mathrm{O}_{2} \mathrm{~S}_{2} \mathrm{Pd}\right]^{+}$. Anal. Found: C, 40.84; H, 2.38; N, 5.88; $\mathrm{S} 6.73 \%$. Calc. for $\mathrm{C}_{32} \mathrm{H}_{26} \mathrm{Cl}_{7} \mathrm{~N}_{4} \mathrm{O}_{2} \mathrm{~S}_{2} \mathrm{Pd}: \mathrm{C}$, 40.34; H, 2.75; N, 5.88; S, 6.73\%. Yield: (0.12g, 68\%).

\section{Synthesis of $\left[\mathrm{Pt}(\operatorname{tenz})_{2} \mathrm{Cl}_{2}\right] \cdot 2 \mathrm{H}_{2} \mathrm{O}(7)$}

$\left[\mathrm{PtCl}_{2}\left(\mathrm{CH}_{3} \mathrm{CN}\right)_{2}\right](0.05 \mathrm{~g}, 0.10 \mathrm{mmol})$ was added to a solution of tioconazole $(0.082 \mathrm{~g}, 0.20 \mathrm{mmol})$ in mixture of acetone and acetonitrile $(15 \mathrm{~mL})$ under reflux during $4 \mathrm{~h}$ at $\mathrm{RT}$ a yellow solid precipitated from the solution. UV-Vis-NIR $v\left(\mathrm{~cm}^{-1}\right): v_{1}=27024$. FT-IR (ATR, $\left.v \mathrm{~cm}^{-1}\right): v(\mathrm{O}-\mathrm{H}) 3444, v(\mathrm{C}=\mathrm{N}) \quad 1588, v(\mathrm{C}-\mathrm{O}-\mathrm{C})$ 1083, v(C-S) 732. ${ }^{1} \mathrm{H}$ NMR (CDCl 3 , 300MHz): 8.24 (2H, s, CH, Ha), $7.65(2 \mathrm{H}, \mathrm{d}, \mathrm{CH}, \mathrm{Hf}), 7.47-7.38(4 \mathrm{H}, \mathrm{m}$, $\mathrm{CH}, \mathrm{Hc}, \mathrm{h}, \mathrm{j}), 7.23$ (2H, m, CH, Hg), 7.17 (2H, m, CH, Hb), 6.88 (2H, d, CH, Hk), 4.97 (2H, dd, CH, He), 4.33 $\left(2 \mathrm{H}, \mathrm{d}, \mathrm{CH}_{2}, \mathrm{Hi}\right), 4.28\left(4 \mathrm{H}, \mathrm{m}, \mathrm{CH}_{2}, \mathrm{Hd}\right), 2.06\left(6 \mathrm{H}, \mathrm{s}, \mathrm{CH}_{3}, \mathrm{Hl}\right) \cdot{ }^{13} \mathrm{C} \mathrm{NMR}\left(\mathrm{CDCl}_{3}, 75 \mathrm{MHz}\right.$ assignments by HSQC): 139.84 (C1), 134.88 (C7), 134.36(C6), 134.16 (C9), 134.65 (C13), 129.77 (C8), 129.64 (C14), 129.46 (C10), 128.40 (C11), 128.26 (C15), 127.02 (C16), 125.06 (C3), 121.37 (C2), 75.78 (C5), 63.53 (C12), 51.43 (C4). MS-ESI ${ }^{+}(\mathrm{m} / \mathrm{z}) 970\left[\mathrm{C}_{32} \mathrm{H}_{26} \mathrm{Cl}_{6} \mathrm{~N}_{4} \mathrm{O}_{2} \mathrm{~S}_{2} \mathrm{Pt}\right]^{+}$. Anal. Found: C, 35.72; H, 2.06; N, 5.48; $\mathrm{S} 5.17 \%$. Calc. for $\mathrm{C}_{32} \mathrm{H}_{30} \mathrm{Cl}_{8} \mathrm{~N}_{4} \mathrm{O}_{4} \mathrm{~S} 2 \mathrm{Pt}$ : C, 35.67; H, 2.81; N, 5.20; S, 5.95\%. Yield: $(0.07 \mathrm{~g}, 61 \%)$.

\section{Synthesis of $\left[\operatorname{Pd}(\operatorname{tenz})_{2}(\mathrm{OAc})_{2}\right](8)$}

$\left[\mathrm{Pd}(\mathrm{OAc})_{2}\right](0.026 \mathrm{~g}, 0.12 \mathrm{mmol})$ was added to a solution of tioconazole $(0.1 \mathrm{~g}, 0.25 \mathrm{mmol})$ in acetone $(15 \mathrm{~mL})$ during $24 \mathrm{~h}$ at RT a beige solid precipitated from the solution. UV-Vis-NIR $v\left(\mathrm{~cm}^{-1}\right)$ : $v_{1}=27683 . \%$. FT-IR (ATR, $\left.v \mathrm{~cm}^{-1}\right): v(\mathrm{C}=\mathrm{N}) 1588, v_{\mathrm{as}}(\mathrm{COO}) 1632, v_{\mathrm{s}}(\mathrm{COO}) 1354 v(\mathrm{C}-\mathrm{O}-\mathrm{C}) 1089, v(\mathrm{C}-\mathrm{S})$ 733. ${ }^{1} \mathrm{H}$ NMR $\left(\mathrm{CDCl}_{3}, 300 \mathrm{MHz}\right): 7.67(2 \mathrm{H}, \mathrm{s}, \mathrm{CH}, \mathrm{Ha}), 7.42(2 \mathrm{H}, \mathrm{d}, \mathrm{CH}, \mathrm{Hf}), 7.35-7.30(4 \mathrm{H}, \mathrm{m}, \mathrm{CH}, \mathrm{Hh}, \mathrm{j})$, $7.17(2 \mathrm{H}, \mathrm{d}, \mathrm{CH}, \mathrm{Hc}), 6.96(2 \mathrm{H}, \mathrm{m}, \mathrm{CH}, \mathrm{Hg}), 6.77(2 \mathrm{H}, \mathrm{d}, \mathrm{CH}, \mathrm{Hk}), 6.73(2 \mathrm{H}, \mathrm{m}, \mathrm{CH}, \mathrm{Hb}), 4.85(2 \mathrm{H}, \mathrm{dd}, \mathrm{CH}$, $\mathrm{He}), 4.40\left(2 \mathrm{H}, \mathrm{d}, \mathrm{CH}_{2}, \mathrm{Hi}\right), 4.24\left(4 \mathrm{H}, \mathrm{d}, \mathrm{CH}_{2}, \mathrm{Hi}\right), 4.09-3.88\left(4 \mathrm{H}, \mathrm{m}, \mathrm{CH}_{2}, \mathrm{Hd}\right)$, ), $1.94\left(6 \mathrm{H}, \mathrm{s}, \mathrm{CH}_{3}, \mathrm{Hl}\right) .{ }^{13} \mathrm{C}$ $\mathrm{CDCl}_{3}, 75 \mathrm{MHzassignments} \mathrm{by} \mathrm{HSQC):178.09} \mathrm{(C17),} 138.55$ (C1), 135.29 (C6), 133.64(C7), 133.42 (C9), 133.35 (C13), 129.79 (C8), 128.73 (C16) ,128.63 (C14), 128.53 (C10), 128.53 (C11), 127.71 (C15), 124.03 $\begin{array}{lllllllllll}(\mathrm{C} 3), & 119.57 & \text { (C2), } 75.89 & \text { (C5), } 63.75 & \text { (C12), } 52.44 & \text { (C4), } 23.66 & \text { (C18). } & \text { MS-ESI }^{+} & \text {(m/z) } & 940\end{array}$ $\left[\mathrm{C}_{34} \mathrm{H}_{29} \mathrm{Cl}_{6} \mathrm{~N}_{4} \mathrm{O}_{4} \mathrm{~S}_{2} \mathrm{Pd}\right]^{+}$. Anal. Found: C, 43.35; H, 2.81; N, 5.99; S 5.77\%. Calc. for $\mathrm{C}_{36} \mathrm{H}_{32} \mathrm{Cl}_{6} \mathrm{~N}_{4} \mathrm{O}_{6} \mathrm{~S}_{2} \mathrm{Pd}: \mathrm{C}$, 43.24; H, 3.23; N, 5.60; S, 6.41. Yield: (0.089g, 69\%).

\section{X-ray crystallographic study}

Diffraction intensity patterns from single crystals of compounds $\mathbf{4}$ and $\mathbf{5}$ were collected on a SMART APEX I diffractometer (Bruker AXS) equipped with a CCD-detector and using graphite monochromated Mo $\mathrm{K} \alpha(\lambda=0.71073 \AA)$ radiation source. APEX2 v2012.10.0 (Bruker, 2012) package was used for data collection and data integration. Absorption corrections were applied using analytical procedure. The structures were 
solved by direct methods using the package SHELXS-2012 and refined with an anisotropic approach for nonhydrogen atoms using the SHELXL-2014/7 program. All hydrogen atoms attached to C atoms were positioned geometrically as riding on their parent atoms, with

$\mathrm{C}-\mathrm{H}=0.93-0.99 \mathrm{~A}$ and $\mathrm{Uiso}(\mathrm{H})=-1.2 \mathrm{Ueq}(\mathrm{C})$ for aromatic and methylene groups.[31-33] A summary for data collection and refinements is given in Table 1.

Table 1. Crystallographic data and refinement parameters of compounds $\left[\mathrm{Ni}(\mathrm{tcnz})_{6}\right] \mathrm{Cl}_{2} 4$ and $\left[\mathrm{Ni}(\mathrm{tcnz})_{6}\right] \mathrm{Br}_{2}$ 5.

\begin{tabular}{|c|c|c|}
\hline Compound & 4 & 5 \\
\hline Empirical formula & $\mathrm{C}_{96} \mathrm{H}_{78} \mathrm{Cl}_{20} \mathrm{~N}_{12} \mathrm{NiO}_{12} \mathrm{~S}_{6}$ & $\mathrm{C}_{96} \mathrm{H}_{78} \mathrm{Br}_{2} \mathrm{Cl}_{18} \mathrm{~N}_{12} \mathrm{NiO}_{6} \mathrm{~S}_{6}$ \\
\hline Formula weight $\left(\mathrm{g} \mathrm{mol}^{-1}\right)$ & 2551.77 & 2640.69 \\
\hline Crystal size $(\mathrm{mm})$ & $0.249 \times 0.217 \times 0.168 \mathrm{~mm}$ & $0.357 \times 0.240 \times 0.234 \mathrm{~mm}$ \\
\hline Crystal color & Purple & Purple \\
\hline Crystal system & Trigonal & Trigonal \\
\hline Space group & $\mathrm{R}-3$ & $\mathrm{R}-3$ \\
\hline \multicolumn{3}{|l|}{ Unit cell dimensions } \\
\hline $\mathrm{a}(\AA)$ & $24.6475(9)$ & $24.655(4)$ \\
\hline $\mathrm{b}(\AA)$ & $24.6475(9)$ & $24.655(4)$ \\
\hline $\mathrm{c}(\AA)$ & $15.8565(6)$ & $15.943(3)$ \\
\hline$\alpha\left(^{\circ}\right)$ & 90 & 90 \\
\hline$\beta\left(^{\circ}\right)$ & 90 & 90 \\
\hline$\gamma\left({ }^{\circ}\right)$ & 120 & 120 \\
\hline $\mathrm{V}\left(\AA^{3}\right)$ & $8342.3(7)$ & $8393(3)$ \\
\hline $\mathrm{Z}$ & 3 & 3 \\
\hline $\mathrm{D}_{\text {calc }}\left(\mathrm{g} / \mathrm{cm}^{3}\right)$ & 1.524 & 1.567 \\
\hline$\mu\left(\mathrm{mm}^{-1}\right)$ & 0.831 & 1.492 \\
\hline $\mathrm{F}(000)$ & 3894 & 3858 \\
\hline $\begin{array}{l}\text { Temp }(\mathrm{K}) \\
\text { Completeness }\end{array}$ & $\begin{array}{l}298(2) \\
99.7 \%\end{array}$ & $\begin{array}{l}298 \\
99.6 \%\end{array}$ \\
\hline $\mathrm{R}_{\text {int }}$ & 0.0641 & 0.0729 \\
\hline $\mathrm{R}(\mathrm{I}>2 \sigma(\mathrm{I}))$ & 0.0906 & 0.0785 \\
\hline $\mathrm{R}_{\mathrm{w}}(\mathrm{I}>2 \sigma(\mathrm{I}))$ & 0.2764 & 0.2213 \\
\hline $\mathrm{S}$ & 1.027 & 1.025 \\
\hline
\end{tabular}

\section{In vitro cytotoxic activity determination Cell culture}

HCT-15 (colon), HeLa (cervix uterine), MCF-7 (breast) and PC-3 (prostate) human carcinoma cell lines were acquired from ATCC (American Tissue Culture Collection) and maintained in incubation at $310 \mathrm{~K}$ and 5\% $\mathrm{CO}_{2}$ with RPMI (GIBCO ${ }^{\circledR}$, Invitrogen corporation) supplemented with $10 \%$ BFS (GIBCO ${ }^{\circledR}$, Invitrogen corporation), $1 \%$ L-glutamine and $1 \%$ penicillin/streptomycin. Experiments were performed with cells within at least 5 passages from each other. All cells were split when around $80-95 \%$ confluence was reached using $0.25 \%$ trypsin/EDTA.

\section{In vitro growth inhibition assay}

After placing $2 \times 10^{4}$ cells/well in 96-well microplate (Costar $\left.{ }^{\circledR}\right)$ with $300 \mathrm{lL}$ capacity and allowed to attach incubating at $310 \mathrm{~K}$ for $48 \mathrm{~h}, \mathrm{HCT}-15$ (colon), HeLa (cervix-uterine), MCF-7 (breast) and PC-3 (prostate) human carcinoma cells were treated with the $\mathrm{Ni}^{\mathrm{iI}}, \mathrm{Pd}^{\mathrm{II}}$, and $\mathrm{P}^{\mathrm{II}}$ complexes. The metal complexes (cisplatin and tioconazole control was added to the plates to act as a positive and comparative control) were tested in $5 \%$ DMSO and in a physiological solution to $0.9 \% \mathrm{NaCl}$ to give a $1 \mathrm{mM}$ stock solution. Two rows 
free of drug solution acted as the $100 \%$ cell survival control. Sonication was sometimes used to facilitate complete dissolution. Serial dilutions were carried out to give final screening, concentrations of the coordination compounds of 400, 200, 20, 2 and $0.2 \mu \mathrm{M}$ (final concentration of DMSO of $0.5 \%(\mathrm{v} / \mathrm{v})$ ). Aliquots of $50 \mu \mathrm{L}$ of these solutions were added to the wells (in triplicate) already containing $150 \mu \mathrm{L}$ of media, so that the final concentrations were $0.01,0.1,1,10$, and $100 \mu \mathrm{g} / \mathrm{mL}$ (final concentration of DMSO of $0.125 \%(\mathrm{v} / \mathrm{v}))$. The cells were exposed to the complex for $24 \mathrm{~h}$, which then was removed and the cells washed with washing media followed by the addition of $200 \mu \mathrm{L}$ of fresh RPMI media. Then the cells were incubated for $72 \mathrm{~h}$ of recovery time. The remaining biomass was then estimated by the sulforhodamine B assay [34-36] (SRB assay). The four screening concentrations were used in an initial test of activity. The selected complexes were then tested for half maximal inhibitory concentration $\left(\mathrm{IC}_{50}\right)$ values determination. Each assay was done in triplicate. $\mathrm{IC}_{50}$ values were obtained from plots of $\%$ cell survival against $\log$ of the drug concentration.

\section{Results and discussion}

A series of coordination compounds of tioconazole with nickel(II), palladium(II) and platinum(II) were synthesized. The geometry depends of the metal ion, with nickel(II) the octahedral compounds $\mathbf{1 - 5}$ were obtained, for palladium and platinum(II) compounds 6-8 square planar arrangement is preferred. The structures of the coordination compounds shown in Fig. 1 are based on their spectroscopic characterization, elemental analyses, molar conductivity, magnetic susceptibility and by X-ray diffraction, when suitable crystals of the coordination compound were obtained.

\section{Spectroscopic characterization}

The IR spectra of the complexes present a characteristic band of the $v(\mathrm{C}=\mathrm{N})$ vibration from the tioconazole ligand, which is shifted to $1586-1589 \mathrm{~cm}^{-1}$, compared with free ligand $\left(1562 \mathrm{~cm}^{-1}\right)$, indicating that the metal ion is coordinated through the imidazolic nitrogen atom. The band associated to the v(C-S) vibration in the tcnz, at $733 \mathrm{~cm}^{-1}$, remains in the same region, $733-737 \mathrm{~cm}^{-1}$, as the sulphur atom does not participate as coordination site. Compound 1 present a broad band at $3324 \mathrm{~cm}^{-1}$ associated to the $v(\mathrm{O}-\mathrm{H})$ vibration of the coordinated water molecule, while for compounds $\mathbf{2 , 3}$ and $\mathbf{7}$ a broad band in the region 3128$3444 \mathrm{~cm}^{-1}$ was assigned to the water molecules of crystallization. The nitrato compound 2 showed three bands associated to the $\mathrm{NO}_{3}$ group, at $1468 \mathrm{~cm}^{-1} v_{\text {as }}\left(\mathrm{NO}_{3}\right), 1306 \mathrm{~cm}^{-1} v_{\mathrm{s}}\left(\mathrm{NO}_{3}\right)$ and $1235 \mathrm{v}(\mathrm{N}=\mathrm{O}) \mathrm{cm}^{-1}$, where the $\Delta v\left(v_{\text {as }}-v_{\mathrm{s}}\right)=162 \mathrm{~cm}^{-1}$ indicating a bidentate coordination mode.[37] Compound 3 presents two intense bands at $1558 \mathrm{~cm}^{-1}, v_{\text {as }}(\mathrm{COO})$, and at $1408 \mathrm{~cm}^{-1} v_{\mathrm{s}}(\mathrm{COO})$, with a $\Delta v\left(v_{\mathrm{as}}-v_{\mathrm{s}}\right)=150 \mathrm{~cm}^{-1}$, characteristic of a bridging coordination mode, [37-40] while for compound $\mathbf{8}, v_{\text {as }}(\mathrm{COO})$ was assigned at $1632 \mathrm{~cm}^{-1}$ and $v_{\mathrm{s}}(\mathrm{COO})$ at $1354 \mathrm{~cm}^{-1}$, with a $\Delta v\left(v_{\mathrm{as}}-v_{\mathrm{s}}\right)=278 \mathrm{~cm}^{-1}$, characteristic of a monodentate carboxylate coordinated to the metal ion.

The solid state electronic spectra (UV-Vis-NIR) for the nickel(II) compounds correspond to an octahedral geometry for the metal atom. For the $\left[\mathrm{Ni}(\operatorname{tcnz})_{3} \mathrm{Br}_{2}\left(\mathrm{H}_{2} \mathrm{O}\right)\right] \mathbf{1},\left[\mathrm{Ni}(\operatorname{tcnz})_{2}\left(\mathrm{NO}_{3}\right)_{2}\right] \cdot \mathrm{H}_{2} \mathrm{O} 2$ and $\left[\mathrm{Ni}(\mathrm{tcnz})_{2}(\mathrm{OAc})_{2}\right]_{2} \cdot 3 \mathrm{H}_{2} \mathrm{O} 3$ compounds, the corresponding electronic transitions were observed: $v_{1}$ ${ }^{3} \mathrm{~T}_{2}(\mathrm{~F}) \leftarrow{ }^{3} \mathrm{~A}_{2 \mathrm{~g}}(\mathrm{~F})\left(8665,8936\right.$ and $\left.8970 \mathrm{~cm}^{-1}\right), v_{2}{ }^{3} \mathrm{~T}_{1 \mathrm{~g}}(\mathrm{~F}) \leftarrow{ }^{3} \mathrm{~A}_{2 \mathrm{~g}}(\mathrm{~F})\left(14993,15553\right.$ and $\left.15417 \mathrm{~cm}^{-1}\right)$ and $v_{3}$ ${ }^{3} \mathrm{~T}_{1 \mathrm{~g}}(\mathrm{P}) \leftarrow{ }^{4} \mathrm{~A}_{2 \mathrm{~g}}(\mathrm{~F})\left(24918,25477\right.$ and $\left.25409 \mathrm{~cm}^{1}\right)$, for $\mathbf{1 - 3}$ respectively. Compounds 4 and $\mathbf{5}$, [Ni(tcnz) ${ }_{6} \mathrm{X}_{2}(\mathrm{X}=$ $\mathrm{Cl}$, Br), presented similar spectra, with $v_{1}{ }^{3} \mathrm{~T}_{2}(\mathrm{~F}) \leftarrow^{3} \mathrm{~A}_{2 \mathrm{~g}}(\mathrm{~F})$ at $11060-11000 \mathrm{~cm}^{-1}, v_{2}{ }^{3} \mathrm{~T}_{1 \mathrm{~g}}(\mathrm{~F}) \leftarrow{ }^{3} \mathrm{~A}_{2 \mathrm{~g}}(\mathrm{~F})$ at $17557-17550 \mathrm{~cm}^{-1}$ and $v_{3}{ }^{3} \mathrm{~T}_{1 \mathrm{~g}}(\mathrm{P}) \leftarrow{ }^{4} \mathrm{~A}_{2 \mathrm{~g}}(\mathrm{~F})$ at $25550-25450 \mathrm{~cm}^{-1}$, with a larger 10Dq $\left(c a .11030 \mathrm{~cm}^{-1}\right)$ than those of compounds 1-3 $\left(\mathrm{ca} .8900 \mathrm{~cm}^{-1}\right)$ due to the coordination of six tenz ligands. For compounds $\left[\mathrm{Pd}(\mathrm{tcnz})_{2} \mathrm{Cl}_{2}\right] \mathbf{6},\left[\mathrm{Pt}(\mathrm{tcnz})_{2} \mathrm{Cl}_{2}\right] \cdot 2 \mathrm{H}_{2} \mathrm{O} 7$ and $\left[\mathrm{Pd}(\mathrm{tcnz})_{2}(\mathrm{OAc})_{2}\right] \mathbf{8}$ a broad band centered on 25595, 27024 and $27683 \mathrm{~cm}^{-1}$ respectively, was assigned to the electronic transition ${ }^{1} \mathrm{~A}_{2 \mathrm{~g}} \leftarrow{ }^{1} \mathrm{~A}_{1 \mathrm{~g}}$, for these metal ions in a square planar environment, (Table 2, Fig. SI 16). [39]

\section{${ }^{1} \mathrm{H}$ and ${ }^{13} \mathrm{C}\left\{{ }^{1} \mathrm{H}\right\}$ NMR spectroscopy}

The ${ }^{1} \mathrm{H},{ }^{13} \mathrm{C}$ and HSQC NMR data (Fig. SI 7-15) supported the coordination mode of the tioconazole to the metal ion, where chemical shifts of the signals was observed compared to free ligand, confirming the proposed trans-square planar structures for the diamagnetic coordination compounds 6-8. 
The ${ }^{1} \mathrm{H}$ NMR spectra of the coordination compounds showed a shift for the $(\mathrm{C} 2-\mathrm{H})$ imidazole proton Ha, between the two nitrogen atoms to $8.08 \mathrm{ppm} \mathrm{6,8.24} \mathrm{ppm} 7$ and $7.67 \mathrm{ppm} \mathrm{8}$, the free ligand present this signal in $7.55 \mathrm{ppm}$ [29], confirming the proposed coordination mode via N3 to the metal ion. The compound 8 presented one singlet in $1.94 \mathrm{ppm}$ due to the acetate.

${ }^{13} \mathrm{C}$ NMR $\left\{{ }^{1} \mathrm{H}\right\}$ spectra of coordination compounds exhibited sixteen signals corresponding to tioconazole, the compound 8 presented two more signals $\mathrm{C} 17$ in $23.66 \mathrm{ppm}$ and $\mathrm{C} 18$ in $178.09 \mathrm{ppm}$ for acetate. The $\mathrm{C} 1$ signal was observed in $137.81 \mathrm{ppm}$ for the free ligand, the shifting in coordination compounds were $139.79,139.84$ and $138.55 \mathrm{ppm}$ for $\mathbf{6 , 7}$ and $\mathbf{8}$, respectively.

\section{Magnetic susceptibility and conductivity}

All the effective magnetic moments ( $\mu_{\text {eff }}$ ) for the nickel(II) complexes are in the range 3.20-3.50 $\mathrm{BM}$, these values are within the expected range for this metal center in a $2+$ oxidation state.[42] The conductivity was measured for all compounds, showing good agreement for the neutral compounds with coordinated halides, nitrates or acetates, compounds 1-3 and 6-8, while the cationic compounds 4 and 5 presented a 1:2 conductivity, Table 2 .

Table 2. Electronic transitions, magnetic moments and molar conductivity of tenz coordination compounds with $\mathrm{Ni}^{\mathrm{II}}, \mathrm{Pd}^{\mathrm{II}}$ and $\mathrm{Pt}^{\mathrm{II}}$.

\begin{tabular}{|l|l|l|l|l|l|l|}
\hline & Coordination compound & $\mu_{\text {eff }}(\mathrm{BM})$ & $\Lambda_{\mathrm{M}}{ }^{\mathrm{a}}$ & $v_{1}\left(\mathrm{~cm}^{-1}\right)$ & $v_{2}\left(\mathrm{~cm}^{-1}\right)$ & $v_{3}\left(\mathrm{~cm}^{-1}\right)$ \\
\hline $\mathbf{1}$ & {$\left[\mathrm{Ni}(\mathrm{tcnz})_{3} \mathrm{Br}_{2}\left(\mathrm{H}_{2} \mathrm{O}\right)\right]$} & 3.40 & 33.9 & 8665 & 14993 & 24918 \\
\hline $\mathbf{2}$ & {$\left[\mathrm{Ni}(\mathrm{tcnz})_{2}\left(\mathrm{NO}_{3}\right)_{2}\right] \cdot \mathrm{H}_{2} \mathrm{O}$} & 3.50 & 13.2 & 8936 & 15553 & 25477 \\
\hline $\mathbf{3}$ & {$\left[\mathrm{Ni}(\mathrm{tcnz})_{2}(\mathrm{OAc})_{2}\right] \cdot 3 \mathrm{H}_{2} \mathrm{O}$} & 3.20 & 10.4 & 8970 & 15417 & 25409. \\
\hline $\mathbf{4}$ & {$\left[\mathrm{Ni}(\mathrm{tcnz})_{6}\right] \mathrm{Cl}_{2}$} & 3.14 & $231.3^{*}$ & 11060 & 17557 & 25550 \\
\hline $\mathbf{5}$ & {$\left[\mathrm{Ni}(\mathrm{tcnz})_{6}\right] \mathrm{Br}_{2}$} & 3.01 & $225.1^{*}$ & 11000 & 17550 & 25450 \\
\hline $\mathbf{6}$ & {$\left[\mathrm{Pd}(\mathrm{tcnz})_{2} \mathrm{Cl}_{2}\right]$} & - & 4.26 & 25595 & - & - \\
\hline $\mathbf{7}$ & {$\left[\mathrm{Pt}(\operatorname{tcnz})_{2} \mathrm{Cl}_{2}\right] \cdot 2 \mathrm{H}_{2} \mathrm{O}$} & - & 1.55 & 27024 & - & - \\
\hline $\mathbf{8}$ & {$\left[\mathrm{Pd}(\mathrm{tcnz})_{2}(\mathrm{OAc})_{2}\right]$} & - & 1.71 & 27683 & - & - \\
\hline
\end{tabular}

${ }^{\mathrm{a}} \Lambda_{\mathrm{M}}$, molar conductance $\left(\mu \mathrm{S} \mathrm{cm} \mathrm{cm}^{-1}\right)$ for $1 \times 10^{-3} \mathrm{M}$ solutions in acetone (no-electrolyte $=0-99 \mu \mathrm{S} \mathrm{cm}{ }^{-1}$ ), and acetonitrile* (electrolyte $1: 2=220-300 \mu \mathrm{S} \mathrm{cm}^{-1}$ ) [43] at $293 \mathrm{~K}$.

\section{$\mathrm{X}$-ray diffraction analysis}

$\left[\mathrm{Ni}(\operatorname{tenz})_{6}\right] \mathrm{Cl}_{2} 4$ and $\left[\mathrm{Ni}(\operatorname{tenz})_{6}\right] \mathrm{Br}_{2} 5$

Crystals of $\left[\mathrm{Ni}(\mathrm{tcnz})_{6}\right] \mathrm{Cl}_{2} \mathbf{4}$ and $\left[\mathrm{Ni}(\mathrm{tcnz})_{6}\right] \mathrm{Br}_{2} 5$ were grown from a saturated acetone solution at room temperature. The compounds are isostructural and crystallized in a trigonal system with an R-3 spatial group. The unit cell consists of three molecules. In both compounds the metal ion presents an octahedral geometry, with six tioconazole molecules in the coordination sphere (Fig. 2). The N3-Ni coordination bond length is 2.12(1) $\AA$. The molecules are highly symmetrical with N-Ni-N angles in the range of $89.14-90.82^{\circ}$ for cis-positions and $179.93^{\circ}$ for trans-positions, and. The nickel(II) atom presents a regular octahedral geometry, despite the fact that ticonazole is a bulky ligand. 


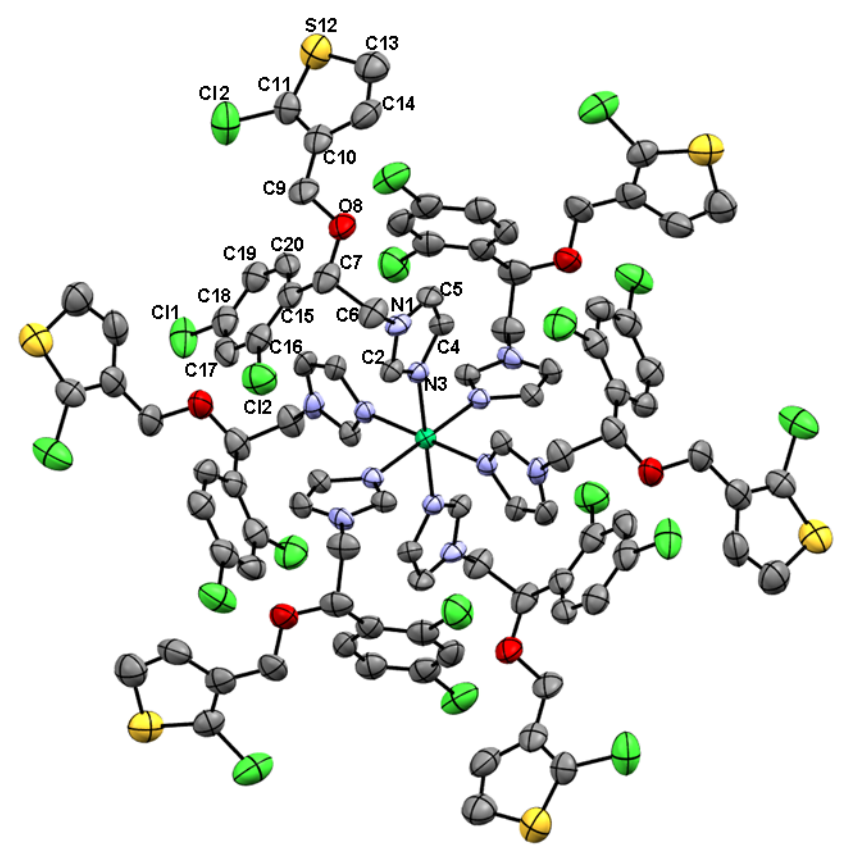

Fig. 2. ORTEP diagram of the $\left[\mathrm{Ni}(\mathrm{tcnz})_{6}\right] \mathrm{Br}_{2} 5$ compound. Displacement ellipsoids are drawn at $30 \%$ probability. $\mathrm{H}$ atoms were omitted for clarity.

Compounds $\mathbf{4}$ and $\mathbf{5}$ present intermolecular hydrogen bonding between the chloro atoms from the thiophen moiety and the methylene hydrogen atoms from neighboring molecules, $\mathrm{C}(9)-\mathrm{H}(9 \mathrm{~B}) \cdots \mathrm{Cl}(2)$, 2.763(4) Å (Fig. 3).

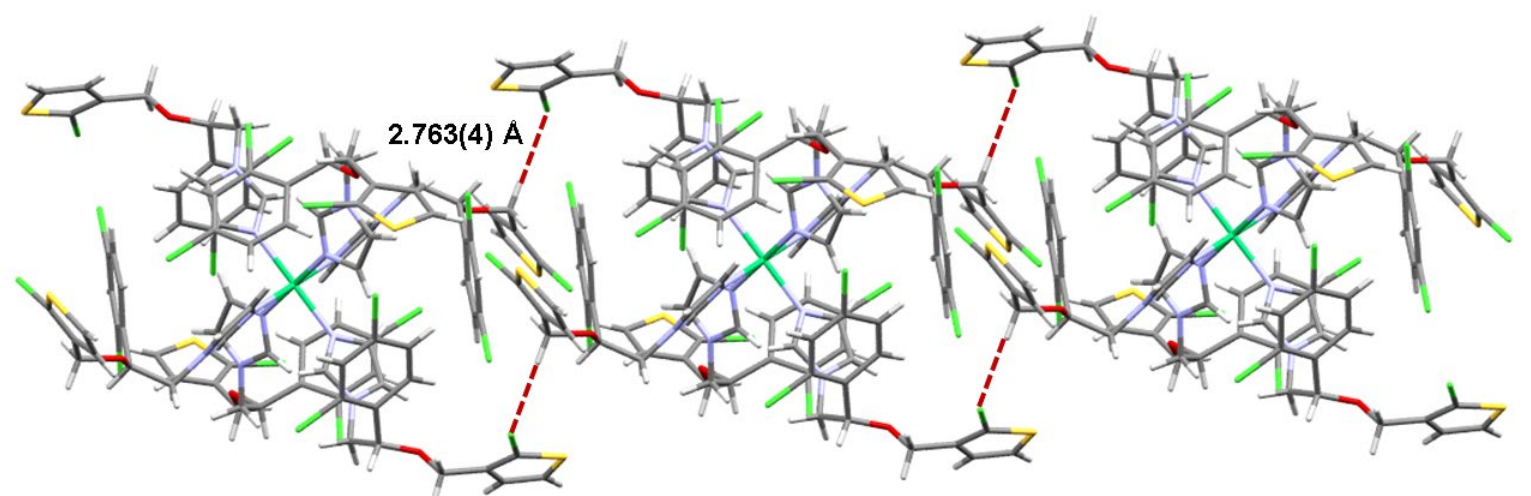

Fig. 3. Intermolecular hydrogen bonding $\mathrm{C}(9)-\mathrm{H}(9 \mathrm{~B}) \cdots \mathrm{Cl}(2)$ in compound $\mathbf{5}$, giving place to a 3D supramolecular arrangement.

The tioconazole ligands are accommodated as a propeller, occupying the six octahedral coordination sites of the nickel(II) atom, giving place to a 3D supramolecular arrangement, stabilized through intermolecular hydrogen bonding and $\pi \cdots \pi$ stacking interactions between the benzene rings of neighboring moelcules, with a distance of $c a$. $C(17) \cdots \pi$ of $3.482 \AA$, from the ring centroid to the $\mathrm{C}(17)$ aromatic carbon, as shown in Fig. 4, [44]. 


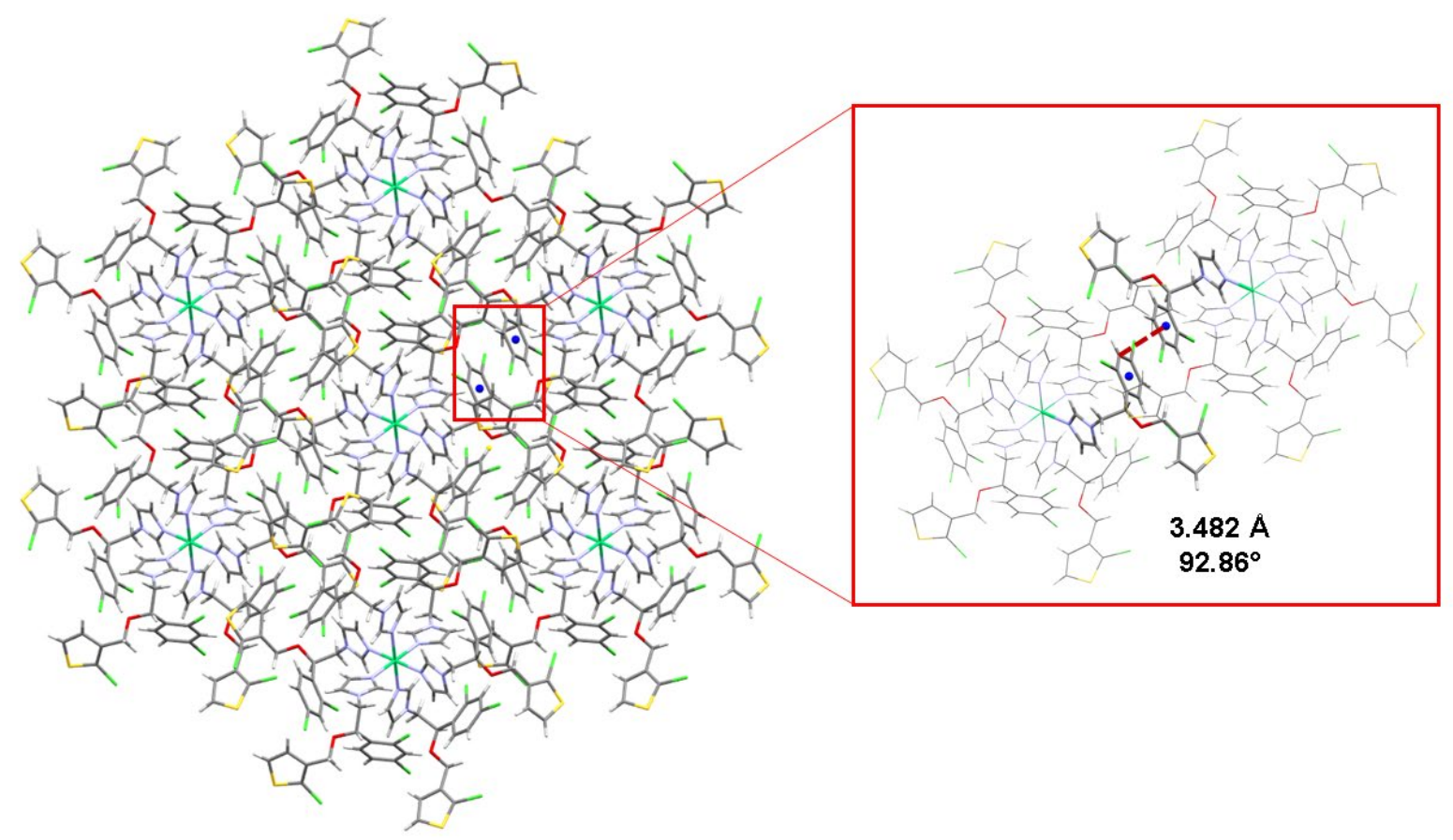

Fig. 4. Supramolecular arrangement stabilized though hydrogen bonding and $\pi \cdots \pi$ stacking in compound 5 .

\section{Cancer cell growth inhibition}

The in vitro cytotoxic activity of the coordination compounds in the human cancer cell lines; HCT15 (colon adenocarcinoma), HeLa (breast adenocarcinoma), MCF-7 (breast) and PC-3 (prostate) was investigated, using cisplatin as reference. The $\mathrm{IC}_{50}$ value $(\mu \mathrm{g} / \mathrm{mL})$ indicates the amount of drug necessary to inhibit $50 \%$ of the growth of cancer cells, after $24 \mathrm{~h}$ of exposition (table 2). The free ligand was not active under these conditions.

In previous work with coordination compounds of imidazole and benzimidazole derivatives it was found that the copper(II) tetrahedral compounds, with coordinated halides, were the most active.[45-47] With the analogous ligand clotrimazole, the tetrahedral nickel(II) coordination compounds presented moderate cytotoxic activity in vitro and the octahedral complexes were not active.[47] Interestingly, for the tioconazole copper(II) octahedral compound a significant cytotoxic activity was observed.[29]

In the present work the nickel(II) octahedral compounds presented cytotoxic activity. The $\left[\mathrm{Ni}(\mathrm{tcnz})_{6}\right] \mathrm{Cl}_{2}$ 4, without halogens in the coordination sphere, showed moderate activity against HCT-15, $\mathrm{HeLa}$, and PC-3; while the $\left[\mathrm{Ni}(\mathrm{tcnz})_{2}\left(\mathrm{NO}_{3}\right)_{2}\right] \cdot \mathrm{H}_{2} \mathrm{O} 2$ compound, presented the best activity in HeLa and MCF7 cell lines, and the $\left[\mathrm{Ni}(\operatorname{tcnz})_{3} \mathrm{Br}_{2}\left(\mathrm{H}_{2} \mathrm{O}\right)\right] \mathbf{1}$ compound showed moderate activity in PC-3, followed by compounds 2, 4 and 5. The palladium(II) and platinum(II) compounds did not presented any significant activity, Table 2. The stability of the studied compounds was determined in DMSO solution and all of them were stable up to 24 hours (Fig. S17). 
Table 3. Cell-growth inhibitory assay results. $\mathrm{IC}_{50}$ value $(\mu \mathrm{g} / \mathrm{mL})$ for $\mathrm{Ni}^{\mathrm{II}}, \mathrm{Pd}^{\mathrm{II}}$ and $\mathrm{Pt}^{\mathrm{II}}$. tioconazole coordination compounds (1-3 and 6-8).

\begin{tabular}{|l|l|l|l|l|l|}
\hline & & \multicolumn{4}{|c|}{ Human carcinoma cell line } \\
\hline & Coordination compound & HCT-15 & HeLa & MCF-7 & PC-3 \\
\hline $\mathbf{1}$ & {$\left[\mathrm{Ni}(\mathrm{tcnz})_{3} \mathrm{Br}_{2}\left(\mathrm{H}_{2} \mathrm{O}\right)\right]$} & 46.35 & 12.15 & 14.26 & 11.43 \\
\hline $\mathbf{2}$ & {$\left[\mathrm{Ni}(\mathrm{tcnz})_{2}\left(\mathrm{NO}_{3}\right)_{2}\right] \cdot \mathrm{H}_{2} \mathrm{O}$} & 18.50 & 7.39 & 12.53 & 16.74 \\
\hline $\mathbf{3}$ & {$\left[\mathrm{Ni}(\mathrm{tcnz})_{2}(\mathrm{OAc})_{2}\right] \cdot 3 \mathrm{H}_{2} \mathrm{O}$} & 323.19 & 32.70 & NA & 31.15 \\
\hline $\mathbf{4}$ & {$\left[\mathrm{Ni}(\mathrm{tcnz})_{6}\right] \mathrm{Cl}_{2}$} & 14.02 & 12.33 & 101.12 & 11.67 \\
\hline $\mathbf{5}$ & {$\left[\mathrm{Ni}(\mathrm{tcnz})_{6}\right] \mathrm{Br}_{2}$} & 303.27 & 10.12 & 15.36 & 16.15 \\
\hline $\mathbf{6}$ & {$\left[\mathrm{Pd}(\mathrm{tcnz})_{2} \mathrm{Cl}_{2}\right]$} & NA & 15.67 & 51.27 & NA \\
\hline $\mathbf{7}$ & {$\left[\mathrm{Pt}(\mathrm{tcnz})_{2} \mathrm{Cl}_{2}\right] \cdot 2 \mathrm{H}_{2} \mathrm{O}$} & ND & ND & ND & ND \\
\hline $\mathbf{8}$ & {$\left[\mathrm{Pd}(\text { tcnz })_{2}(\mathrm{OAc})_{2}\right]$} & NA & NA & NA & NA \\
\hline & Cisplatin & 8.25 & 5.55 & 1.3 & 3.83 \\
\hline
\end{tabular}

$\mathrm{ND}=$ not determined $\mathrm{NA}=$ not active

\section{Conclusions}

Coordination compounds with tioconazole and $\mathrm{Ni}^{\mathrm{II}}, \mathrm{Pd}^{\mathrm{II}}$, and $\mathrm{Pt}^{\mathrm{II}}$ were synthesized and fully characterized. The coordination compounds stabilized octahedral (1-5) and square planar (6-8) geometries, depending on the metal ion. Despite that tioconazole is a bulky ligand it can be accommodated in a propeller arrangement, occupying the six octahedral coordination sites of a nickel(II) atom. The crystallographic arrangements of compounds $\mathbf{4}$ and $\mathbf{5}$ were stabilized through hydrogen bonding and $\pi \cdots \pi$ stacking interactions.

The octahedral nickel(II) compounds showed moderate cytotoxic activity (HeLa), the IC $_{50}$ increased upon coordination to the metal ion when compared to the inactive free ligand, which can be related to the nature and the geometry of the metal ion, as the square planar platinum(II) and palladium(II) complexes did not presented any significant activity.

\section{Supplementary Information}

CCDC No. 1581442 for $\left[\mathrm{Ni}(\mathrm{tcnz})_{6}\right] \mathrm{Cl}_{2}$ and 1581443 for $\left[\mathrm{Ni}(\mathrm{tcnz})_{6}\right] \mathrm{Cl}_{2}$ contains the supplementary crystallographic data. These data can be obtained free of charge from The Cambridge Crystallographic Data Centre via www.ccdc.cam.ac.uk/data request/cif. Supplementary Information associated with this article can be found in the online version.

\section{Acknolwedgements}

C. C.-L. thanks DGAPA-UNAM for the posdoctoral fellowship. R. N.-P. thanks CONACyT for MSc scholarship. Financial support of CONACYT CB2012-17885 and DGAPA-UNAM IN224516 grants is acknowledged. We thank Patricia Fierro for her technical support.

\section{References}

1. Castiñeiras, A.; Fernández-Hermida, N.; García-Santos, I.; Gómez-Rodríguez, L. Dalton Trans. 2012, 43, 13486-13495

2. Macombera, L.; Hausinger, R. P. Metallomics, 2011, 3, 1153-1162 
3. Ragsdale, S. W. Curr. Op. Chem. Biol. 1998, 2, 208-215

4. Boer, J. L.; Mulrooney, S. B.; Hausinger, R. P. Arch. Biochem. Biophys. 2014, 544, 142-152

5. Tottaa, X.; Papadopouloua, A.A.; Hatzidimitrioua, A. G.; Papadopoulos, A.; Psomas, G. J. Inorg. Biochem. 2015, 145, 79-93

6. Chohan, Z. H.; Arif, M.; Shafiq, Z.; Yaqub, M.; Supuran, C. T. J. Enz. Inhib. Med. Chem. 2006, 21, 95-103

7. Williams, C. J.; Whitehouse, J. M. A., Br. Med. J. 1979, 1, 1689-1691

8. Langer, C.J.; Gadgeel, S. M.; Borghaei, H.; Papadimitrakopoulou, V. A.; Patnaik, A.; Powell, S. F.; Gentzler, R. D.; Martins, R.G.; Stevenson, J. P.; Jalal, S. I.; Panwalkar, A.; Yang, J. C.; Gubens, M.; Sequist, L. V.; Awad, M. M.; Fiore, J.; Ge, Y.; Raftopoulos, H.; Gandhi, L. Lancet Oncol. 2016, 11, $1497-1508$

9. Williams, K.J.; Picus, J.; Trinkhaus, K.; Fournier, C. C.; Suresh, R.; James, J. S.; Tan, B. R. HPB 2010, 6, 418-426

10. Alcindor, T.; Beauger, N. Curr. Oncol. 2011, 1, 18-25

11. Eddings, D.; Barnes, C.; Gerasimchuk, N.; Durham, P.; Domasevich, K. Inorg. Chem. 2004, 43, 3894-3909

12. Jiang, Y.; Shan, S.; Gan, T.; Zhang, X.; Lu, X.; Hu, H.; Wu, Y.; Sheng, J.; Yang, J. Biomed. Rep. 2014, $2,893-897$

13. Xiang, K.; Hai-Hua, X.; Hai-Qin, S.; Xia-Bin, J.; Le-San, Y.; Ruo-Gu, Q. Cancer Biol. Med. 2015, $12,362-374$

14. Wong, E.; Giandomenico, C. M. Chem. Rev. 1999, 99, 2451-2466

15. Gao, E.; Liu, L.; Zhu, M.; Huang, Y.; Guan, F.; Gao, X.; Zhang, M.; Wang, L. Zhang, W.; Sun, Y. Inorg. Chem. 2011, 50, 4732-4741

16. Jahromi, E. Z.; Divsalar, A.; Saboury, A. A.; Khaleghizadeh, S.; Mansouri-Torshizi, H.; Kostova, I. J. Iran Chem. Soc. 2016, 13, 967-989

17. Sharma, N. K.; Ameta, R. K.; Singh, M. Inter. J. Med. Chem. 2016, 2016, 1-10

18. Bingchang, Z.; Haiqing, L.; Qinjuan, X.; Lirong, L.; Bing, Z. Oncotarget. 2017, 8, 13620-13631

19. Hartmann, J. T.; Lipp, H. Expert. Opin. Pharmacother. 2003, 6, 889-901

20. Sabokrouh, A.; Vaisi-Raygani, A.; Goodarzi, M. T.; Khatami, S.; Taghizadeh-Jahed, M.; Shahabadi, N.; Lakpour, N.; Shakiba, Y. Avicenna. J. Med. Biotechnol. 2015, 7, 50-56

21. O'Brien, M. E.; Szczesna, A.; Karnicka, H.; Zatloukal, P.; Eisen, T.; Hartmann, W.; Kasan, P.; Longerey, B.; Lefresne, F. Annals of Oncology. 2004, 15, 921-927

22. Abu-Surrah, A. S.; Abu-Safieh, K. A.; Ahmad, I. M.; Abdalla, M. Y.; Ayoub, M. T.; Qaroush, A. K.; Abu-Mahtheieh, A. M. Europ. J. Med. Chem. 2010, 45, 471-475

23. Abu-Surrah, A. S.; Al-Sa'doni, H. H.; Abdalla, M. Y. Cancer Therapy 2008, 6, 1-10

24. Garoufis, A.; Hadjikakou, S. K.; Hadjiliadis, N. Coord. Chem. Rev. 2009, 253, 1384-1397

25. Hernández, W.; Paz, J.; Carrasco, F.; Vaisberg, A.; Spodine, E.; Manzur, J.; Hennig, L.; Sieler, J.; Blaurock, S.; Beyer, L. Bioinorg. Chem. \& Appl. 2013, 2013, 1-12

26. Ali, M. A.; Mirza, A. H.; Butcher, R.; Tarafder, M. T. H.; Keat, T. B.; Ali, A. M. J. Inorg. Biochem. 2002, $92,141-148$

27. Kljun, J.; Scott, A. J.; Rizner, T. L.; Keiser, J.; Turel, I. Organometallics. 2014, 33, 1594-1601

28. Singh, K.; Kumar, Y.; Puri, P.; Kumar, M.; Sharma, C. Eur. J. Med. Chem. 2012, 52, 313-321

29. Crisóstomo-Lucas, C.; García-Holley, P.; Hernández-Ortega, S.; Sánchez-Bartéz, F.; Gracia-Mora, I.; Barba-Behrens, N. Inorg. Chim. Acta. 2015, 438, 245-254

30. Mathews, C. J.; Smith, P. J.; Welton, T. J. Mol. Catal. A: Chem. 2004, 214, 27-32

31. Sheldrick, G. M. Acta Cryst. 2008, A64, 112

32. Clark, R. C.; Reid, J. S. Acta Cryst. 1995, A51, 887

33. Hübschle, C. B.; Sheldrick, G. M.; Dittrich, B. J. Appl. Cryst. 2011, 44, 1281-1284

34. Rubinstein, L. V.; Shoemaker, R. H.; Paull, K. D.; Simon, R. M.; Tosini, S.; Skehan, P.; Scudiero, D. A.; Monks, A.; Boyd, M. R. J . Nat. Cancer Inst. 1990, 82, 1113-1117

35. Skehan, P.; Storeng, R.; Scudiero, D.; Monks, A.; McMahon, J.; Vistica, D.; Warren, J. T.; Bokesch, H.; Kenney, S.; Boyd, M. R. J Nat. Cancer Inst. 1990, 82, 1107-1112

36. Alley, M. C.; Scudiero, D. A.; Monks, A.; Hursey, M. L.; Czerwinski, M. J.; Fine, D. L.; Abbott, B. J.; Mayo, J. G.; Shoemaker, R. H.; Boyd, M. R. Cancer Res. 1988, 48, 589-601 
37. Nakamoto, K. Infrared and Raman Spectra of Inorganic Coordination Compounds, John Wiley \& Sons, 1986

38. Bourke, J. P.; Cannon, R. D.; Grinter, G.; Jayasooriya, U. A. Spectrochim. Acta. 1993, 49A, 685-690

39. Pal, S.; Gohdes, J. W.; Wilisch, W. C. A.; Armstrong, W. H. Inorg. Chem. 1992, 31, 713-716

40. Gielen, M. Appl. Organomet. Chem. 2002, 16, 481-494

41. Lever, A. B., Inorganic Electronic Spectroscopy, 2da. Ed., Amsterdam, Elsevier, 1984

42. Sigel, H. Metal Ions in Biological Systems, Marcel Dekker, 1975

43. Angelici, A. J., Synthesis an technique in inorganic chemistry, 2da., ed., USA, University Science Books, 1986

44. Janiak, J. C. J. Chem. Soc. Dalton Trans. 2000, 2000, 3885-3896

45. Sánchez-Guadarrama, O.; López-Sandoval, H.; Sánchez-Bartez, F.; Gracia-Mora, I.; Hopfl, H.; Barba-Behrens, N. J. Inorg. Biochem. 2009, 103, 1204-1213

46. López-Sandoval, H.; Londono-Lemos, M. E.; Garza-Velasco, R.; Poblano-Meleléndez, I.; GranadaMacías, P.; Gracia-Mora, I.; Barba-Behrens, N., J. Inorg. Biochem. 2008, 102, 1267-1276

47. Betanzos-Lara, S.; Gracia-Mora, I.; Granada-Macías, P.; Flores-Álamo, M.; Barba-Behrens, N. Inorg. Chim. Acta. 2013, 397, 94-100 